\title{
Regressive Prediction Approach to Vertical Handover in Fourth Generation Wireless Networks
}

\author{
Abubakar M. Miyim, Mahamod Ismail, Rosdiadee Nordin \& M. Taha Ismail \\ Department of Electrical, Electronics \& Systems Engineering, \\ Universiti Kebangsaan Malaysia, 43600 Bangi Selangor, Malaysia \\ Email: ammiyim@eng.ukm.my
}

\begin{abstract}
The over increasing demand for deployment of wireless access networks has made wireless mobile devices to face so many challenges in choosing the best suitable network from a set of available access networks. Some of the weighty issues in $4 \mathrm{G}$ wireless networks are fastness and seamlessness in handover process. This paper therefore, proposes a handover technique based on movement prediction in wireless mobile (WiMAX and LTE-A) environment. The technique enables the system to predict signal quality between the UE and Radio Base Stations (RBS)/Access Points (APs) in two different networks. Prediction is achieved by employing the Markov Decision Process Model (MDPM) where the movement of the UE is dynamically estimated and averaged to keep track of the signal strength of mobile users. With the help of the prediction, layer-3 handover activities are able to occur prior to layer-2 handover, and therefore, total handover latency can be reduced. The performances of various handover approaches influenced by different metrics (mobility velocities) were evaluated. The results presented demonstrate good accuracy the proposed method was able to achieve in predicting the next signal level by reducing the total handover latency.
\end{abstract}

Keywords: Delay; DRIMA; LTE-Advanced; MDP; RDPD; WiMAX.

\section{$1 \quad$ Introduction}

As diverse wireless communication services proliferate and anytime-anywhere communication is becoming a norm in mobile communication, mobile devices should achieve seamless operation when roaming within wireless networks even under high mobility environment. Emerging broadband wireless air interface specifications, such as IEEE $802.16 \mathrm{~m}$ and Third Generation Partnership Project (3GPP) Long Term Evolution (LTE)-Advanced, are designed with stringent requirements on interruption time during handover in order to provide quality of service (QoS) for current and future mobile wireless applications. To provide seamless services in such a wireless mobile communication environment, delay or disruption in dealing with mobility must be minimized. However, offering services such as VoIP on IEEE 802.16 networks [1],[2] is considered problematic due to very long handover latency.

Received September $30^{\text {th }}, 2013$, Revision March $10^{\text {th }}$, 2014, Accepted for publication April $1^{\text {st }}, 2014$.

Copyright $\odot 2014$ Published by ITB Journal Publisher, ISSN: 2337-5787, DOI: 10.5614/itbj.ict.res.appl.2014.8.1.3 
IEEE 802.16 handover delay comprises of cells scan latencies from neighbouring cells, handover message exchange (HOME) and network re-entry. However, minimization procedures of network re-entry can occur by handover optimization process as defined in [2]. Nevertheless, resolution of address and registration of networks makes Mobile IP handover latency to be relatively lengthy when compared to layer-2 latency. Consequently, this handover latency is sometimes not acceptable for real-time applications. Additionally, the difference that exists between the interfaces of IEEE802.16e MAC and that of 3GPP handover techniques leads to adverse effect on the overall handover latency [3]. The UE through the cross-layer mobility protocols detect that it has changed from the current subnet to a new subnet provided by the new AP and relevant information [3]-[5]. Link layer information, like received signal strength (RSS) reduces the delay in mobility detection for Cross-layer mobility management protocols. Although UE utilizes signal of layer 2 (L2) trigger to concurrently prompt layer-3 with those of layer- 2 handover, it is equally capable of giving early notification for possible change of point of attachment (PoA) to the access network.

Many researchers have confirmed that approaches which are prediction-based do reduce handover latency by predicting the next position of the UE having considered the patterns of its previous movement [6]-[8]. In [7], a mobility model that takes cognisance not only of historical records, but also stochastic behaviours of UE to predict their future locations was proposed. Although some models seem to work quite well in environments with non-stationary data, but the intricacies of the algorithms and indirect computation time often make them unworkable.

In this paper, a simple regressive differential prediction and decision (RDPD) method that accurately trigger handover based on mobility prediction of two wireless mobile networks (WiMAX and LTE-A) has been proposed. The mobility prediction is set to be achieved using a novel dynamic regressive integrated moving average (DRIMA) model. By keeping trace of the RSS between the mobile user equipment (UE) and radio base stations (RBS's), dynamically estimating regression movement and averaged to predict the next signal level. Total handover latency can be reduced with layer-3 handover occurring prior to layer-2 handover taking place with the assistance of prediction.

The rest of this paper is organized as follows. Related works is being discussed in Section 2. Section 3 describes the mathematical formulation of the proposed prediction technique while the fourth section discusses the new Prediction Technique. Simulation scenarios and parameters considered for assessing the 
efficiency of the novel prediction as well as the analysis of the results are given in Section 5. Section 6 concludes the paper.

\section{Related Work}

Mobile User Equipment (UE) performs handover when some signal parameters of the serving Radio Base Station (RBS) to which the UE is connected drops below a predefined level. When the UE disconnects from the serving RBS and subsequently start negotiating connection with a new RBS, a time interval is recorded between signal disruption with the serving RBS and the setting up of a new connection with the target RBS and this scheme is called hard handover. This minimal sudden breakage in communication can degrade the QoS. Consequently, the UE can connect simultaneously to more than one RBS while it performs handover and this type of handover is called soft handover. The minimization of break in hard handover or that of an overhead generated as a result of the handover procedure is easily achieved by handover prediction. Another purpose for the handover prediction is to optimize an admission control as presented in [3],[4]. The utilization of handover prediction for reservation of resources for admission control is also presented in [5]. Two schemes of admission control were proposed by the paper to optimize the utilization of dedicated bandwidth. The effectiveness of the prediction is considered in [16] for the minimization of the power consumption in ad-hoc networks. Reduction of the consumed power is accomplished by suspending the handover until the UE moves nearer to the target station and such prediction is executed based on the UE's movement history [2]. In [7], the paper presents advanced algorithms for the prediction of user's location where the precise location of the user is required and that implies the use of localization equipment such as the Global Positioning System (GPS) [9]. Methods of filtering handover prediction were evaluated in [10], where the authors compared the efficiency of handover prediction using Grey Algorithm (GA), Kalman, Fourier and Particle filtering of Received Signal Strength Indication (RSSI) values. The results showed a better performance (about $80 \%$ of successful handover prediction hit ratio) for no filtering and Grey filtering techniques. The Grey filtering technique which was evaluated and analyzed in [11], shows reduction of a number of handovers performed by the Grey prediction Algorithm thereby making positive impact on the performance of the entire network.

Two handover prediction approaches comprising of network-centric (cellular) and user-centric (mobile terminal) were investigated in [12]. The Cell approach predicts a number of users in the cell while user approach utilizes a mobility prediction for handover estimation. Furthermore, the paper gave a summary of the advantages of both approaches and their suitability for utilization in different situations. The authors meanwhile, discuss a new proposed mechanism 
for resource allocation that demonstrates a better performance with user approach to minimize handover failures. On the other hand, the cell approach improve cell blocking probability. Therefore, handover prediction could be defined as a process where the next target RBS is predicted while considering a prior knowledge of the parameters of the target RBS that can enable the fast handover with minimal interruption. Besides, it can reduce the MAC management overhead due to optimization of the list of neighbourhood station for scanning. The prediction can be based on several approaches as was earlier enumerated in the previous literatures.

However, designing an efficient vertical handover algorithm has always been a critical issue in heterogeneous wireless networks that tend to provide seamless services. It is always tasking to design an efficient handoff algorithm for heterogeneous environments, to support seamless communication services. In most literatures [13]-[15], fuzzy logic was applied in handover decision making process because of its inherent strength in providing solution to imprecision problems. In [15], a proposed handoff decision that uses principle of fuzzy logic to lessen unnecessary handoff in the homogeneous cellular system was implemented. While [15] utilizes speed and traffic load to decide the RSS hysteresis values, using adaptive fuzzy based vertical handoff algorithm, on the other hand [16] provides a multi-criteria decision-making algorithm based on fuzzy theory for multiple access network selection. Furthermore, the complex nature of the system has to be resolved before being utilized. Fuzzy logic principle was not used to decide the handover hysteresis values as found in [15], but rather it was used to evaluate the intensity of the input parameters.

Goyal, et al. in their proposal [17] adopted a dynamic decision model in choosing the "best" network at the "right and best" time to handover. The proposed model decides the best among the available networks using dynamic factors like the Received Signal Strength (RSS) and velocity of the mobile UE simultaneously with static factors such as monetary cost, bandwidth availability and power consumption. However, the model did not only meet the individual user needs, but reduce the unnecessary handovers which influences improvement on the entire performance of the system. In [18], Abd El-Fadeel, et al. proposed a Multi-attributes SINR Based Vertical Handoff algorithm for next generation heterogeneous wireless networks with Predictive SINR, using GM $(1,1)$. It combines the effects of SINR, required bandwidth, traffic cost, utilization from participating access networks and user preference to make handover decision QoS aware which becomes difficult to achieve with traditional RSS as a vertical handover criteria.

In [19], a trajectory-aware handoff algorithm that is based on location, speed, delay and received signal strength (RSS) of mobile UE was proposed. Two 
types of velocity; tangential and radial velocities were used in the algorithm having radial velocity of UE being considered in the handover decision making and ignoring tangential velocity of UE during handover initiation. More so, least square line method is applied to RSS of UE to check the Ping-Pong effects. A dwell timer based vertical handover scheme was suggested by Ayyappan, et al. in [20] to ameliorate handoff delay (service quality degradations), packet losses, decreased throughput and network disconnection for Cellular networks and WLAN integrated networks. This handover algorithm was found to be useful in reducing the handover delay and maximizing the throughput by choosing the optimum dwell time. Another method of vertical handover scheme described in [21] is based on the mobility prediction of the UE. In contrast with the existing schemes, the proposed approach considers the movement velocity and direction of the UE, and Received Signal Strength (RSS) for accurate prediction of the UE's movement toward a specific access point. The scheme utilizes IPv6 to Solicit for Router Proxy (RtSolPr) and Proxy Router Advertisement (PrRtAdv) messages with the PAR and obtains the IP address and subnet prefix information of the NAR associated to the nearby base station. Computer simulation reveals that the proposed scheme significantly outperforms the existing scheme in terms of handover success rate and messaging overhead, especially when the velocity of UE is relatively high and the number of APs is large.

Tawil, et al. [22] proposed a Simple Additive Weighting (SAW) method for vertical handover decision scheme to improve the service mobility for use in a distributed manner under heterogeneous environments. In [23] and [24] an algorithm that uses Hidden Markov Model (HMM) predictor was adapted to accurately estimate the next location visited by a Mobile UE, given current and historical movement information. The algorithm achieved an overall 62\% increase in throughput when compared to other Vertical Handoff Decision Algorithms. The aim was to reduce the overload and the processing-delay in the mobile terminal by calculating the handover metrics to select the Target Networks. On the other hand, in [25], an overall gain function was proposed to make the right decision based on cost of usage, bandwidth, power consumption as well as dynamic factors like the Received Signal Strength (RSS), Velocity and Position of mobile UE. Furthermore, Xie, et al. [26] proposed a new algorithm for heterogeneous wireless networks (cellular and WLANs). Cost function, based on the probabilities of blocking and dropping call rates were calculated for cellular network and WLAN using the channel-guard call admission method.

Chandavarkar, et al. [27] have proposed an Interface selection algorithm based on coverage area, available bandwidth and power consumption of the mobile UE. Singh, et al. [28] have proposed a vertical handoff decision algorithm that 
determines whether a vertical handoff should be initiated and dynamically selects the optimum network connection from the available access network technologies to continue with an existing service or begin another service entirely.

\section{Vertical Handover Prediction Formulation}

The RSS of multi-model is a time series during the handover and its current value has intrinsic relation with historical values. DRIMA model is based on mathematical statistics and random process, which exert the current value and the historical value of the time series to predict the future RSS value. This model draws on the advantages of regression analysis and considers the prediction. Based on the value of current RSS and the values of previous RSS, the proposed approach adopt DRIMA model to predict the value of the next RSS.

The structure of this model is formulated by defining the sequence; $\mathrm{S}(\mathrm{t})=$ $\{\mathrm{s}(0)(1), \mathrm{s}(0)(2), \ldots \ldots, \mathrm{s}(0)(\mathrm{N})\}$ to represent $\mathrm{N}$ sampled RSS. Considering this sequence to be the initial random data and carries noise, then further process has to be applied on them. The accumulated values generated (AVG) are to be used in processing the initial data.

$$
\mathrm{s}^{(1)}(\mathrm{K})=\sum \mathrm{s}^{(0)}(\mathrm{k}) ;(\mathrm{k}=1,2, \ldots, \mathrm{N})
$$

By employing linear dynamic modelling to approximate $1^{\text {st }}$ order AVG sequence $\mathrm{ds}^{(1)} / \mathrm{d}+\mathrm{as}{ }^{(1)}=\mathrm{b}$, Where $\mathrm{a}$ and $\mathrm{b}$ - coefficients of the differential equation whose solution is given as

$$
\mathrm{s}^{(1)}(\mathrm{N}+1)=\left[\mathrm{s}^{(0)}(1)-\mathrm{b} / \mathrm{a}\right] \mathrm{e}^{-\mathrm{aN}}+\mathrm{b} / \mathrm{a}
$$

$a$ and $b$ are coefficients that could be determined by least square approximation.

In this algorithm, the time series is composed of RSS, and error term is the error of the previous prediction. Some of the common methods like least squares, moment estimation; maximum likelihood estimation and so on could be used to determine the parameters of DRIMA. In this paper, we adopt least squares to determine the parameter. The vector representing these coefficients is:

$$
\begin{gathered}
\hat{\mathrm{a}}=[\mathrm{a}, \mathrm{b}] \mathrm{T}(\mathrm{BTB})-1 \mathrm{BTYN}, \text { where } \mathrm{B}=[\text { Matrix }] \\
\text { and } \Upsilon \mathrm{N}=[\mathrm{N}(0)(2), \mathrm{N}(0)(3), \ldots, \mathrm{N}(0)(\mathrm{N})] \mathrm{T} \\
\hat{\mathrm{s}}(0)(\mathrm{N}+1)=[\mathrm{s}(0)(1)-\mathrm{b} / \mathrm{a}] \mathrm{e}-\mathrm{aN} \mathrm{x}(1-\mathrm{ea})
\end{gathered}
$$


and, so the predictive RSS $=\hat{s}^{(0)}(\mathrm{N}+1)$. In a typical heterogeneous network environment, it is possible to specify constant with values different from the characteristics of certain network type.

\subsection{Evaluation of Vertical Handover Decision}

The quantitative evaluation of handover decision (QDV) has become necessary when three metrics are considered for integration. For the handover decision to be optimized, weight of every metric of a candidate network has to be adjusted to conform to the relative priority of other attributes in different networks. This will show the dynamism of the constantly changing qualitative evaluations under predictable wireless environment that could raise the dominant-difference among candidate networks.

$$
\begin{aligned}
& \overline{Q D V^{R S S}}=\frac{1}{(N-1)} \sum_{i=1}^{N} Q D V^{R S S} \\
& \delta^{R S S}=\sqrt{\frac{1}{N-1}} \sum_{i=1}^{N}\left(Q D V_{i}^{R S S}-\overline{Q D V^{R S S}}\right)^{2}
\end{aligned}
$$

$\overline{Q D V^{R S S}}$ is average QDV of RSS. The smaller the value, the more significant is the RSS factor and the bigger the assigned weight of RSS.

$\delta^{\mathrm{RSS}}$ is standard deviation of QDV of RSS, the larger weight of RSS is assigned, the larger will be the $\delta^{\mathrm{RSS}}$.

Therefore the key factor of RSS is:

$$
\Omega^{R S S}=e^{\left(-Q D V_{i}^{R S S}+\delta^{R S S}\right)}
$$

\section{The Proposed Prediction Technique}

The proposed algorithm uses Markov Decision Process to dynamically predict the UE regressive moving average and to also monitor the network QoS, which eventually decides the possibility of handover triggering and network selection to choose the appropriate access network. It is a fact that handover trigger is associated with both the measured and the predicted RSS, whereas network selection is tied to user preference. This makes the types of service application (real and non-real time) to be in agreement with period of RSS measurements. 


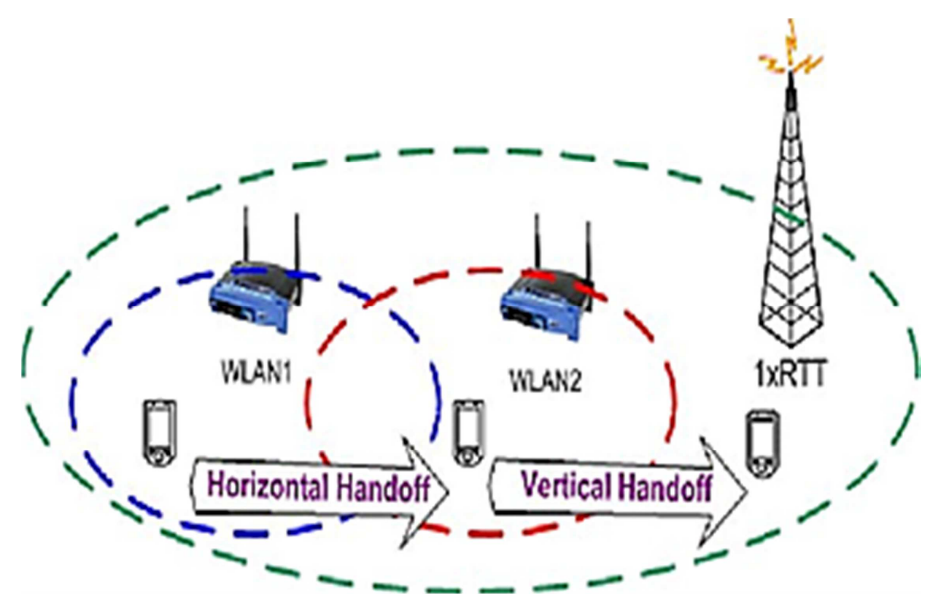

Figure 1 Diagram of a Wireless Heterogeneous Network (HWN).

A typical heterogeneous wireless network (HWN) depicted in Figure 1 is made up of overlay of wireless networks (WLANs and 3GPP) that applies both the vertical and horizontal handover schemes. The UE moves from one location to the other across the overlay network within the coverage area of the network. Making reference to the Figure, we consider a UE in 3GPP network with the Predictive RSS (PRSS) trying to determine the movement of UE towards the target network during response time by comparing the PRSS with the maximum threshold (RSSmax_th_WL/WM). When a UE stays in LTE-A/WiMAX, it begins to sense whether the RSS of the current serving network is less than the minimum threshold (RSSmin_th_WL/WM). Both the upper and lower levels of the computed response time tend to be short for real time applications. This was possible for the handover delay to be minimized; otherwise it will take longer time for non-real applications to execute the handover. Thereafter, the UE cross check to confirm the strength (PRSS) of every target network, when they are being compared to the maximum threshold (RSSmax_th_WL/WM). However, when their merit value surpasses the current serving network, the target networks then become candidate network to be handed over.

DRIMA model is based on mathematical statistics and random process, which exert the current value and the historical value of the time series to predict the next RSS value. The model draws on the advantages of differential regression analysis and considers the prediction errors in calculation process, giving it a smaller prediction error variance and suitable for short term prediction. User equipment (UE) periodically scans the signal frame from the RBS to get the RSS and then computes the parameters of the DRIMA model according to the obtained RSS making possible the prediction of the next RSS for the link layer 
triggering event. During the process of prediction, the mechanism is not only considered for the dependence of signal power value on time series, but also considered as prediction error thereby setting the accuracy of predicted signal high [29]. Based on this idea, the moment parameters of the link layer achieve a pre-set threshold, a messages is sent to activate the network layer handover. However, as the threshold is significantly small, the efficiency of the handover is reduced which in turn causes Ping-Pong with large threshold. The algorithm is based on the predicted RSS value, which sets up a threshold smaller than the threshold of the predicted RSS value the of the activated link layer. So the handover is performed much earlier before the RSS becomes weak to avoid Ping-Pong as depicted in the flow chart of Figure 2.

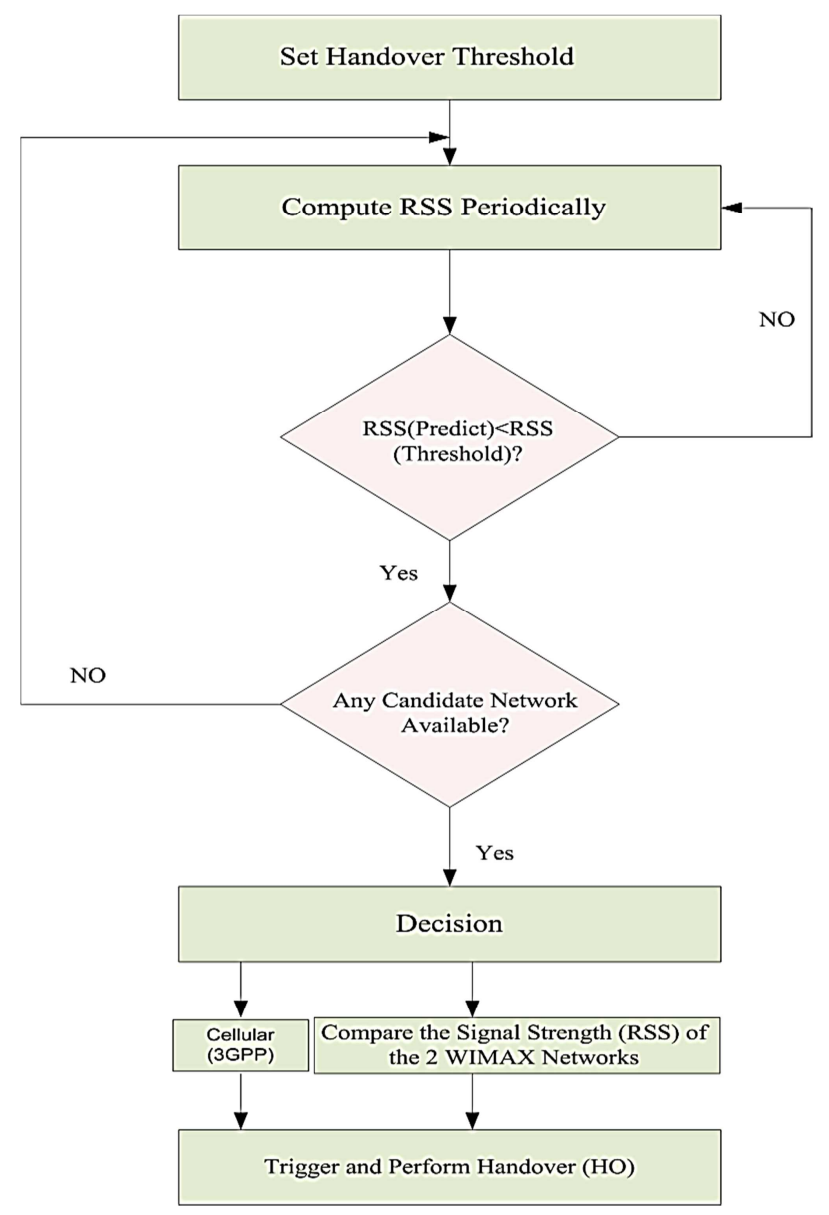

Figure 2 Proposed Vertical Handover Prediction Algorithm. 


\subsection{Efficiency of Prediction}

As the efficiency of the RSS prediction continues to suffer, the impact is being felt by a number of neighbouring RBSs. The proposed scheme has predictive RSS mechanism consisting of two major stages: predictive RSS with hysteresis and that of defining the optimal candidate wireless network in which to hand over. The earlier stage deals with a proposed scheme of polynomial regressionbased prediction of RSS and it is meant to collaborate with a hysteresis for determine/predict if a mobile UE location is closer to or away from the candidate network. For the later stage, it is the handover cost that is to be determined based on the Markov decision process (MDP) method [6] and [7] and the network that has the least handover cost is then selected as the optimal handover candidate network. It is to achieve load balancing and non-dropping probability.

\subsubsection{Predictive RSS Handover}

In trying to avoid the problem of Ping-Pong as a result of unwanted handovers, RSS-based with hysteresis is the scheme considered here. Although mobile UE transmit/receive signals between two thresholds signal levels just to stop the unwanted handovers, they however, suffer from low data bit rates and weak RSS when the received RSS of the serving network is near the lowest threshold value. Such a low data bit rates and weak RSS do cause low utilization and high dropping probability.

In this handover approach, the UE execute handover in overlap areas; thereby causing Ping-Pong effect whenever the UE come into the overlap areas. Thus, this proposal of a predictive RSS-based handover approach is to perform handover at a certain point when the mobile UE predicts that it is getting closer to the candidate network. The advantages of this scheme are: 1) The handover process is capable of performing prior to RSS becoming weak and thus obtains better quality of service (QoS) and higher data rate. 2) Not only unwanted handovers are being avoided, but also minimizes the dropping probability.

\subsubsection{Polynomial Regression-Based Prediction}

There are two approaches to the proposed RSS Based Handover: pre-process and RSS prediction.

Pre-process: In dealing with prediction, the measures of the previous RSSs are important so as to determine the next predictive RSS. To intensify the polynomial regression-based curve fitting, the pre-process of accumulated generating operation, which is based on [10], is adopted to achieve the accuracy 
of the prediction results. The pre-process generates a new data sequence by summation of the previous $n$ data, which are formulated by

$$
S^{\prime}(n)=\sum_{p=1}^{n} S(p)
$$

where, $S(p)$ is the original data sequence, and $S^{\prime}(n)$ is the new sequence after executing the pre-process. The purpose of the pre-process is to smooth the fitting curve. Consequently, the benefit of using a technique based on hysteresis prediction scheme is to avoid unwanted handover, reducing the dropping probability and to obtain high data rates. The implemented weight adjustment algorithm uses back-propagation scheme [29] to predict the RSS in the fourth generation networks as shown in Figure 3, where the predictive RSS was accurate and matches the actual/real RSS values.

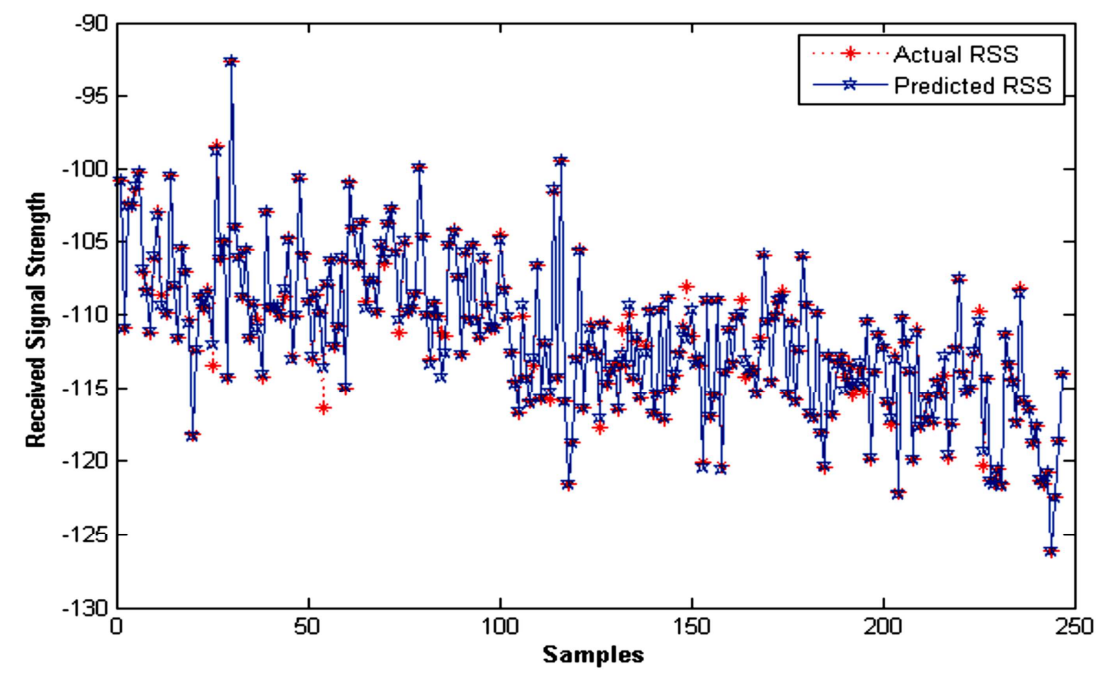

Figure 3 Prediction results with the plot of RSS vs. Samples.

RSS Prediction: After the pre-process step, the new data sequence is used as the input data for curve fitting in the RSS prediction step. The predictive RSS in the new sequence is denoted as PRSS', which is computed by

$$
\text { PRSS }^{\prime}=\mathrm{F}_{\text {predict }}(\mathrm{t}+1)
$$

Since PRSS' $\left(S^{\prime}(n+1)\right)$ has been computed by Eq. (9), the predictive RSS of the original sequence PRSS can be determined by the reverse transformation of Eq. (8) as 


$$
S(n+1)=S^{\prime}(n+1)-S^{\prime}(n)
$$

The approach of polynomial regression-based predictive RSS is demonstrated in Figure 4 as the actual and predictive RSSs of a mobile UE roaming in a heterogeneous wireless network in which only the previous RSSs are adopted for each prediction. From the figure, it is observed that the predictive RSS always predicts accurately, although the actual RSS is at the next point of each peak, which is the most difficult prediction point.

$$
\frac{1}{\Pi} \sum_{x=1}^{\Pi} \frac{\left|P R S S_{x}-R S S\right|}{|R S S|}
$$

\section{$5 \quad$ Simulation}

\subsection{Simulation Parameters}

Two overlapping network technologies (IEEE802.16 and 3GPP) depicted in Figure 1 is considered for the simulation. The bigger coverage represents LTEA network, while the two overlapping circles are WiMAX network topologies. The trajectory of a moving UE is assumed to follow a certain mobility pattern, where the simulation parameters of the mobile user are given in the Table 1. Channel propagation model used is given as: $\operatorname{RSS}(d)[d B m]=\operatorname{Pt}[d B m]-$ $\mathrm{PL}(d)[d B m], \mathrm{Pt}$ - transmit power and PL is the Free space path loss of the link.

Table 1 Simulation Parameters

\begin{tabular}{ccccccccc}
\hline Parameter & $\begin{array}{c}\text { Cell } \\
\text { Radius } \\
(\mathbf{m})\end{array}$ & $\begin{array}{c}\text { Transmit } \\
\text { Power } \\
\text { (Watts) }\end{array}$ & $\begin{array}{c}\text { RSS_min_Th } \\
{[\mathbf{d B m}]}\end{array}$ & $\begin{array}{c}\text { RSS_max_Th } \\
{[\mathbf{d B m}]}\end{array}$ & $\begin{array}{c}\text { UE } \\
\text { speed } \\
{[\mathbf{m} / \mathbf{s}]}\end{array}$ & $\begin{array}{c}\text { Frequency } \\
\text { band } \\
{[\mathbf{G H z}]}\end{array}$ & $\begin{array}{c}\text { Simulation } \\
\text { duration } \\
{[\mathbf{s}]}\end{array}$ & $\begin{array}{c}\text { Fading } \\
(\boldsymbol{\sigma})\end{array}$ \\
\hline 3GPP & 600 & 1 & -100 & -80 & 10 & 2.6 & 100 & 4 \\
WiMAX & 100 & 0.001 & -102 & -84 & 10 & 2.4 & 100 & \\
\hline
\end{tabular}

\subsection{Results and Analysis}

From Figure 4, we can observe that the predictive RSS always predicts accurately, although the actual RSS is at the next point of each peak, which is the most difficult prediction point. Figure 3 displays the vertical handovers of UE in the overlayed network, where the changing curves of current RSSs from RBS and APs vary with the changing location of UE. It can be figured out that the position of AP is almost at the midpoint of the simulation time, but might not necessary be the midpoint of the distance between the RBS and AP as shown; rather AP could be nearer the RBS than to the AP. Therefore in the first handover procedure, the condition of the network for UE outperforms that of the second handover. That is to say that the performance evaluation of the networks in the first handover looks bigger than that of the second handover. 
Figure 4 shows the locations where handovers occur. It illustrates that the proposed algorithm can execute handovers accurately and eliminates the PingPong effect.

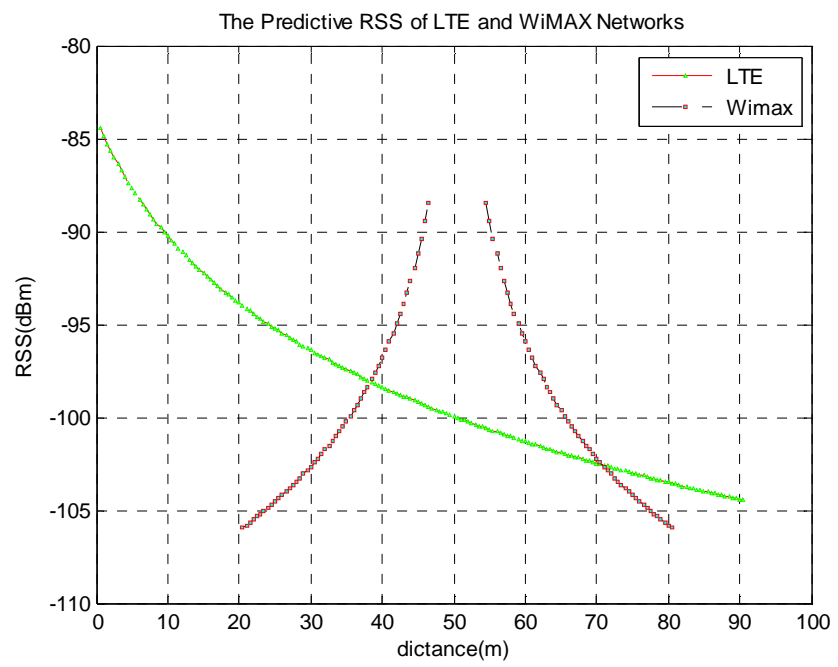

Figure 4 Predictive RSS Handover Curves of UE in (WiMAX \& LTE-A) Networks.

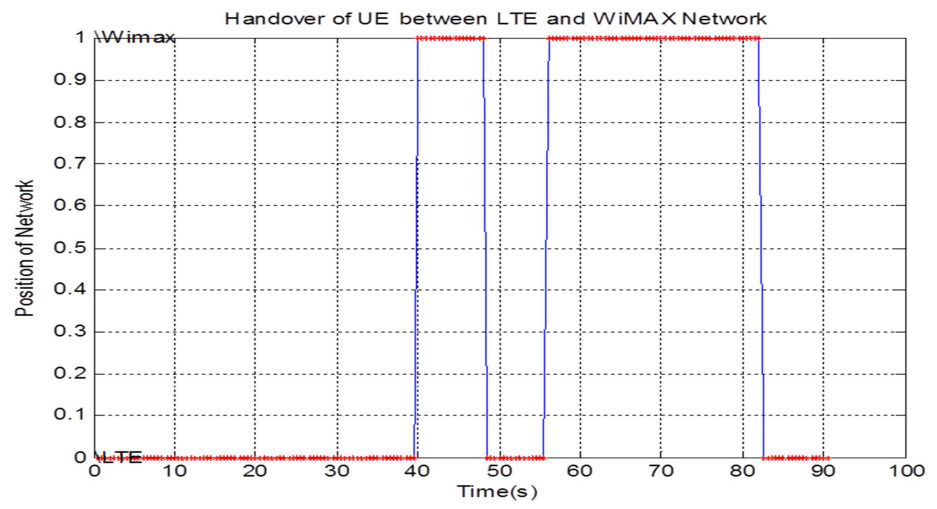

Figure 5 Number of Vertical Handover Occurrences as UE roams the Networks.

However, the UE seems to stays long in the LTE-A network for almost 40s before handing over to the first WiMAX network which took less than 10s to stay in it (i.e., LTE-A/WiMAX). In the second handover between the LTE-A and the second WiMAX (i.e. WiMAX/LTE-A), the period the UE takes to stay 
is less than the first handover (about 20s) before the RSS drops and eventually changes to the LTE-A network again. This phenomenon is demonstrated in Figure 5.

\subsection{Handover Performance}

The results of simulations are presented to show the performance of the proposed MDP-based approach by comparing it to other two methods, fuzzy logic based vertical handover algorithm and the traditional method using the maximum of the PRSS and hysteresis threshold (PRSS+HT) [14],[24]. In assessing the performance of various vertical handover approaches influenced under different mobility velocities ( 5 to $25 \mathrm{~m} / \mathrm{s}$ ), metrics are evaluated and demonstrated in Figures 6 and 7. In Figure 6, PRSS_Poly+MDP yield the least number of vertical handovers under changing velocities, but RSS-T has high number of vertical handovers. Furthermore, the numbers of vertical handovers of all the approaches rise as the velocity increases. The proposed PRSS_Poly+MDP approach results in the few vertical handovers and better resource utilization than RSS-T, RSS-HT, and PRSS_Cost as demonstrated in Figure 7. The performance of PRSS_Poly+MDP is very low, but that of RSS-T and RSS-HT increases as the arrival rate, mobility, or number of nodes increases. The primary reason is that PRSS_Poly+MDP choose the WLAN/WWAN with the lowest cost as the optimal target network to hand over which is determined by MDP analysis.

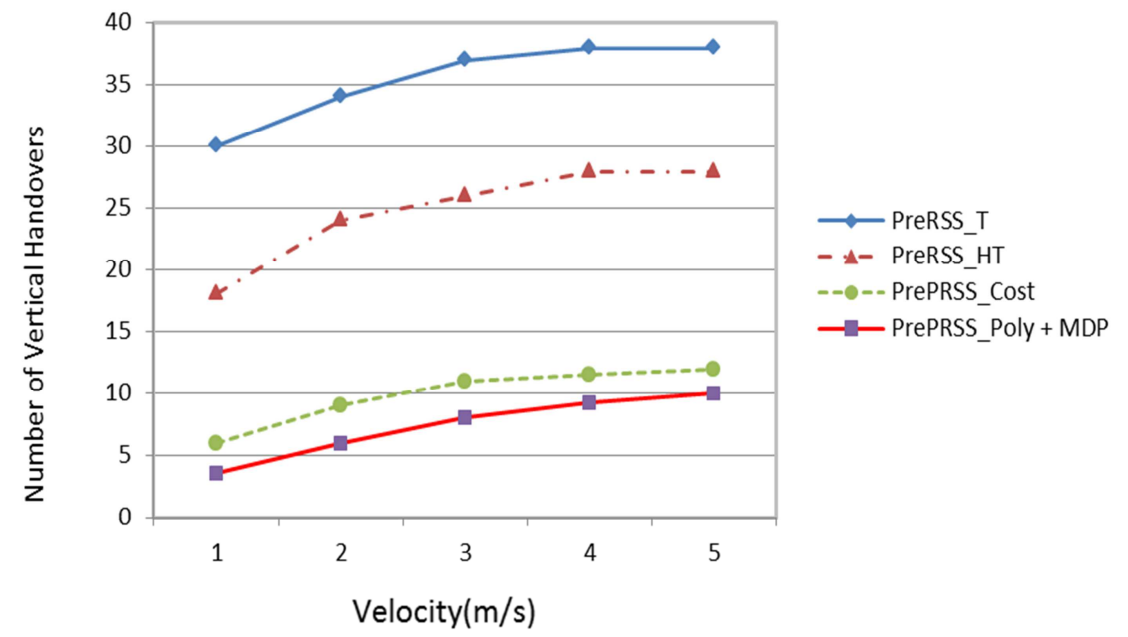

Figure 6 Successful Vertical Handovers under various Velocities. 
The proposed approach could be said to achieve load balancing and avoids unwanted handovers while yielding competitive utilization. As indicated in Figure 7, the average utilization per call in WLAN/3GPP network of all approach has generally improved as the arrival rates keep increasing with the MDP-based having an edge in network utilization than the other methods. Consequently, PRSS_Poly+MDP outperform other approaches of other performance metrics of vertical handover in $4 \mathrm{G}$ wireless networks. Furthermore, the proposed MDP-based scheme has significantly improved the throughput of the TCP.

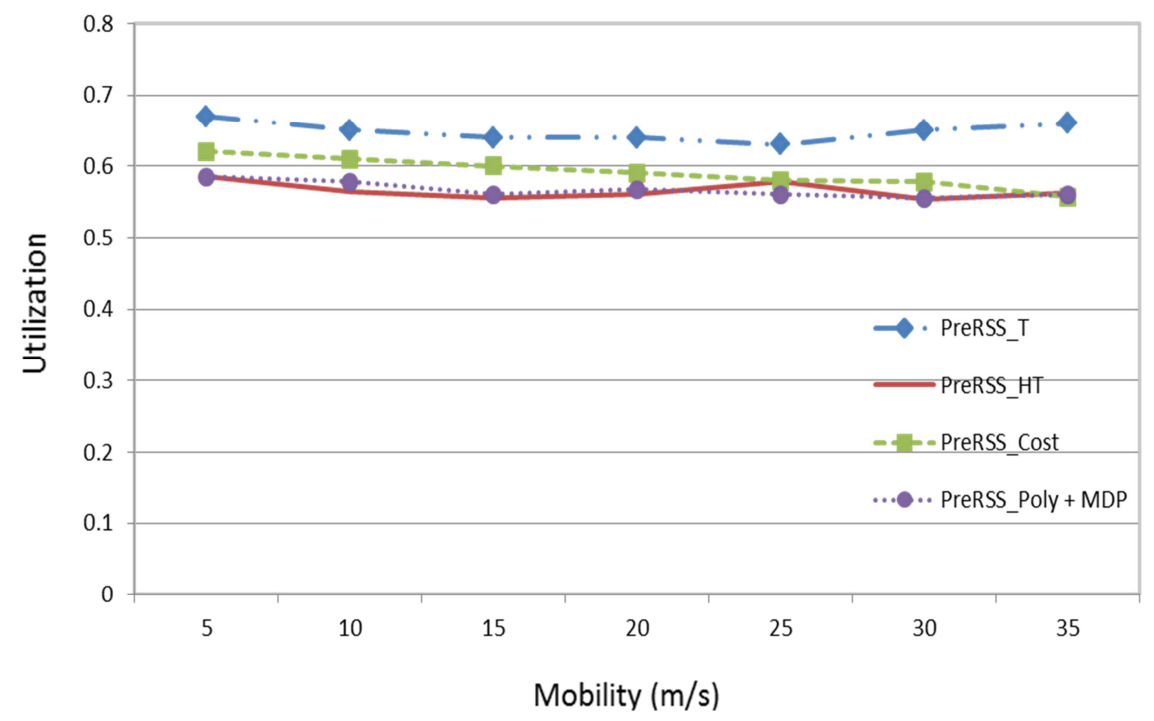

Figure 7 Network Utilization vs. Mobility.

\section{Conclusion}

An intelligent vertical handover algorithm in heterogeneous 4G wireless networks has been presented where the efficient resource utilization of the target network prediction based handover was investigated. The proposed handover scheme takes into cognisance velocity, current RSS and predicted RSS of candidate networks as the network parameters. The algorithm adopts a polynomial regressive predictive RSS, capable of triggering a handover in advance using MDP-based optimal selection. Two approaches were considered; predictive RSS based on polynomial regression to predict if the location of UE is either close or away from the monitored target network. The second approach uses MDP analysis to select the candidate network with the lowest/least handover cost as the optimal network. For the network to accurately trigger 
handover and filter out the unwanted data, a pre-decision process is applied before the handover decision module. The proposed algorithm can save time in choosing the best candidate network among them. The results show that the new technique provides good ground to minimize Ping-Pong in heterogeneous wireless networks which has been executed intelligently to decide the dynamism of the network conditions. It also demonstrated that minimum number of handovers could be obtained at different speeds when the new method was compared with other schemes. In addition, the MDP-based is seen to make good utilization of the resources available when compared to others. The benefit driven from the use of predictive RSS is to assist the mobile UE predict the location of the target RBS/AP to avoid dropping of handover connections and achieve high data bitrate and better transmission quality.

\section{Acknowledgement}

This research was funded by Universiti Kebangsaan Malaysia (UKM) under the grant: DPP-2013-006. The authors would like to thank the sponsor for the financial support towards the publication of this work. Also wish to acknowledge the contributions of the reviewers for their useful advice in an effort to improving the quality of the paper.

\section{Reference}

[1] Liu, T. \& Bahl, P., Mobility Modeling, Location Tracking and Trajectory Prediction in Wireless ATM Networks, IEEE Journal on Selected Areas in Communications, 16(6), pp. 922-936, 1998.

[2] Becvar, Z., Mach, P. \& Simak, B., Improvement of Handover Prediction in Mobile WiMAX by using two Thresholds, Journal of Computer Networks, 55(16), pp. 41-47, 2011.

[3] Martinez-Bauset, J., Gimenez-Guzman, M. \& Pla, J.V., Optimal Admission Control in Multimedia Mobile Networks with Handover Prediction, IEEE Wireless Communication, 15(5), pp. 38-44, 2008.

[4] Triantafyllopoulou, D., Passas, N., \& Kaoxylos, A., Integration of Handover in a Cross-Layer Mechanism for Mobile Multimedia Systems, Proceedings of IEEE International Conference on Communications, Dresden, pp. 4973-4977, June 2009. doi:10.1109/ICC.2009.5199085.

[5] Cheng, X. \& Bi, D., Real-Time Adaptive Link Layer Trigger Based Cross Layer Fast Handoff Mechanism in IEEE802.11 WLANs, Proceedings of the International Conference on Communication Software and Networks, pp. 443-447, 2009.

[6] Lu, L.-L. \& Wu, J.-L.C., Handoff Prediction by Mobility Characteristics in Wireless Broadband Networks, $6^{\text {th }}$ IEEE Symposium on a World of Wireless Mobile and Multimedia Networks, Mannheim, pp. 469-471, June 2005. doi: 10.1109/WOWMOM.2005.49. 
[7] Chakraborty, S., Dong, Y., Yau, D.K.Y. \& Lui, J.C.S., On the Effectiveness of Movement Prediction To Reduce Energy Consumption in Wireless Communication, IEEE Transactions on Mobile Computing, 5(2), pp. 157-169, 2006.

[8] Miyim, A.M., Ismail, M. \& Nordin, R., Prioritized Network-Based Handover Decision in Multi-Access Wireless Networks, Proceedings of Asia-Pacific Conference on Communications, pp. 828-833, 2012.

[9] GPS System Description, Global Positioning System Precise Positioning Service Performance Standard, 2007, www.gps.gov/technical/ps/2007PPS-performance-standard.pdf (12 October 2012).

[10] Bellavista, P., Corradi, A. \& Giannelli, C., Evaluating Filtering Strategies for Decentralized Handover Prediction in the Wireless Internet, $11^{\text {th }}$ IEEE Symposium on Computers and Communications, pp. 167-174, 2006,.

[11] Rezaei, S.S.C. \& Khalaj, B.H., Grey Prediction Based Handoff Algorithm, International Journal of Information and Communication Engineering, 1(7), pp. 321-324, 2005.

[12] Aghalya, S. \& Seethalakshmi, P., An Efficient Decision Algorithm for Vertical Handoff Across 4G Heterogeneous Wireless Networks, International Journal Computer Science and Information Security, October, 2010.

[13] Xia, L., Jiang, L.-G. \& He, C., A Novel Fuzzy Logic Vertical Handoff Algorithm with Aid of Differential Prediction and Pre-Decision Method, Proceedings of IEEE Communications Society, pp. 5665-5670, 2007.

[14] Kunarak, S. \& Suleesathira, R., Predictive RSS with Fuzzy Logic Based Vertical Handoff Algorithm in Heterogeneous Wireless Networks, Proceedings of International Symposium on Communications and Information Technologies, pp. 1235-1240, 2010.

[15] Xia, L., Jiang, L.-G., He, C. \& Hong-Wei, L., An Intelligent Vertical Handoff Algorithm in Heterogeneous Wireless Networks, Proceedings of International Conference on Neural Network and Signal Processing, pp. 550-555, 2008.

[16] Lee, S.K., Sriram, K., Kim, K., Kim, Y.H. \& Golmie, N., Vertical Handoff Decision Algorithms for Providing Optimized Performance in Heterogeneous Wireless Networks, IEEE Transactions on Vehicular Technology, 58(2), pp. 865-881, 2009.

[17] Goyal, P. \& Saxena, S.K., A Dynamic Decision Model for Vertical Handoffs across Heterogeneous Wireless Networks, World Academy of Science, Engineering and Technology, 2(5), pp.1288-1293, 2008.

[18] Abd El-Fadeel, G., El-Sawy, A.E. \& Adib, M.J., Vertical Handoff in Heterogeneous Wireless Networks with Predictive SINR using GM(1,1), $29^{\text {th }}$ National Radio Science Conference (NRSC 2012), Cairo, Egypt, pp.175-184, April 2012. 
[19] Thazin, E. \& Wang F., A Trajectory-Aware Handoff Algorithm Based on GPS Information, SpringerLink, Annals of Telecommunications, 65(7-8), pp. 411-417, 2009.

[20] Ayyappan, K., Dhanraj, R., Dananjayan, P. \& Kumar, R., Dwell Timer Based Vertical Handoff Scheme for Heterogeneous Wireless Networks, MASAUM Journal of Computing, 1(2), 2009.

[21] Jung, B.Y., Choi, M.S., Youn, H.Y. \& Song, O., Vertical Handover based on the Prediction of Mobility of Mobile Node", Proceedings of the $8^{\text {th }}$ IEEE International Conference on Pervasive Computing and Communications Workshops (PERCOM Workshops), pp. 534-539, March 2010. doi:10.1109/PERCOMW.2010.5470496.

[22] Tawil, R., Demerjian, J., Pujolle, G. \& Salazar, O., Processing-Delay Reduction during the Vertical Handoff Decision in Heterogeneous Wireless Systems, Proceedings of the IEEE/ACS International Conference on Computer Systems and Applications, April 2008, pp. 381385. doi:10.1109/AICCSA.2008.4493561.

[23] Zdenek, B., Efficiency of Handover Prediction Based on Handover History, Journal of Convergence Information Technology, 4(4), pp. 4147, 2009.

[24] Osman Gani, M.D., Prediction of State of Wireless Network Using Markov and Hidden Markov Model, Journal of Networks, 4(10), pp. 976984, 2009.

[25] Aghalya, S. \& Sivasubramanian, A., An Optimized Vertical Handoff Decision Algorithm Based on Mobility Prediction in Heterogeneous Wireless Networks, European Journal of Scientific Research, 94(2), pp. 177-185, 2013.

[26] Xie, S. \& Wu, M., Vertical Handoff Decision Algorithm to Minimize the Network Cost, Proceedings of IEEE 4th International Conference on Wireless Communication, Networking and Mobile Computing, Dalian, pp. 1-4, 2008. doi:10.1109/WiCom.2008.1011008.

[27] Chandavarkar, B.R. \& Ram Mohana Reddy, G., Improvement in Packet Drop during handover between WiFi and WiMax, International Conference on Network and Electronics Engineering, IPCSIT 11 IACSIT Press, Singapore, 2011.

[28] Singh, R.K., Asthana, A., Balyan, A., Gupta, S.J. \& Kumar, P., Vertical Handoffs in Fourth Generation Wireless Networks, International Journal of Soft Computing and Engineering (IJSCE) 2(2), 2012.

[29] Amutha, B. \& Ponnavaikko, M., Energy Efficient Hidden Markov Model Based Target Tracking Mechanism in Wireless Sensor Networks, Journal of Computer Science, 5(12), pp. 1085-1093, 2009. 